\title{
Long-term, Real-life, Observational Study in Treating Outpatient Ulcerative Colitis with Golimumab
}

\author{
Antonio Tursi ${ }^{12^{*}}$, Giammarco Mocci ${ }^{3^{*}}$, Walter Elisei ${ }^{4}$, Leonardo Allegretta ${ }^{5}$, Raffaele Colucci ${ }^{6}$, Nicola Della Valle ${ }^{7}$, \\ Antonio de Medici ${ }^{8}$, Roberto Faggiani ${ }^{4}$, Antonio Ferronato ${ }^{9}$, Giacomo Forti ${ }^{10}$, Tiziana Larussa ${ }^{11}$, Roberto Lorenzetti ${ }^{12}$, \\ Francesco Luzza ${ }^{11}$, Antonio Penna ${ }^{13}$, Giuseppe Pranzo ${ }^{14}$, Stefano Rodino ${ }^{15}$, Rodolfo Sacco ${ }^{7}$, Ladislava Sebkova ${ }^{15}$, \\ Costantino Zampaletta ${ }^{16}$, Camilla Graziosi ${ }^{16}$, Marcello Picchio ${ }^{17}$, Irene Maria Bambina Bergna ${ }^{18}$, Giovanni Maconi ${ }^{18}$
}

See Authors' affiliations at the end of the paper.
Received: 30.08 .2021

Accepted: 03.11.2021

\footnotetext{
* A.T. and G.M. equal contribution
}

\begin{abstract}
Background \& Aims: Several studies have found Golimumab (GOL) effective and safe in the short-term treatment of ulcerative colitis (UC), but few long-term data are currently available from real world. Our aim was to assess the long-term real-life efficacy and safety of GOL in managing UC outpatients in Italy.

Methods: A retrospective multicenter study assessing consecutive UC outpatients treated with GOL for at least 3-month of follow-up was made. Primary endpoints were the induction and maintenance of remission in UC, defined as Mayo score $\leq 2$. Several secondary endpoints, including clinical response, colectomy rate, steroid free remission and mucosal healing, were also assessed during the follow-up.

Results: One hundred and seventy-eight patients were enrolled and followed up for a median (IQR) time of 9 (3-18) months (mean time follow-up: $33.1 \pm 13$ months). Clinical remission was achieved in 57 (32.1\%) patients: these patients continued with GOL, but only 6 patients $(3.4 \%)$ were still under clinical remission with GOL at the $42 \mathrm{nd}$ month of follow-up. Clinical response occurred in 64 (36.4\%) patients; colectomy was performed in $8(7.8 \%)$ patients, all of them having primary failure.

Steroid-free remission occurred in 23 (12.9\%) patients, and mucosal healing was achieved in 29/89 (32.6\%) patients. Adverse events occurred in 14 (7.9\%) patients.

Conclusions: Golimumab does not seem able to maintain long-term remission in UC in real life. The safety profile was good.
\end{abstract}

Key words: induction - follow-up - golimumab - remission - treatment - ulcerative colitis.

Abbreviations: AE: adverse event; CRP: C-reactive protein; FC: fecal calprotectin; GOL: golimumab; HR: hazard ratio; IQR: interquartile range; $\mathrm{MH}$ : mucosal healing; s.c.: subcutaneous; TNFa: tumor necrosis factor a; UC: ulcerative colitis.

\section{INTRODUCTION}

Ulcerative colitis (UC) is chronic inflammatory disease of the colon characterized by a complex interaction between genetic and environmental factors [1]. Since it is characterised by a relapsing and remitting course, sometimes it requires an aggressive therapeutic approach in order to prevent complications [2]. Tumor necrosis factor $a$ (TNFa) plays an important role in the pathogenesis of the disease [1], and the introduction of monoclonal anti-TNFa antibodies infliximab and adalimumab has greatly improved our treatment options in UC patients refractory or intolerant to standard treatments $[2,3]$.

Golimumab (GOL) is a subcutaneously (s.c.) administered fully human IgG1 kappa anti-TNF monoclonal antibody [4], already approved in rheumatic disorders [5-10]. PURSUIT trials found s.c. GOL effective in obtaining and maintaining remission in moderate-to-severe UC $[11,12]$, while no effectiveness was found when the drug was administered by the intravenous route [13]. Several real-life studies have been published on patients with UC treated with GOL in daily clinical practice [14-21] and have identified some factors possibly associated with a better response rate to GOL, such as a lack of previous exposure to anti-TNF antibodies and short duration of disease. However, these studies had generally a relatively small sample size, thus limiting the generalizability of their results and, more important, demonstrating a limited follow-up. To date, three studies have had a follow-up longer than 12 months with a probability of persistence in GOL and of maintenance of response at 1 year 
ranging from 60 to $40 \%[19,22,23]$. However, more studies would be useful to confirm these results.

The aim of this study was to assess the long-term efficacy and safety of GOL in treating a large Italian UC outpatient population.

\section{METHODS}

This study consisted of a retrospective, cohort, observational study on UC outpatients unresponsive to standard treatments and treated with s.c. GOL in 15 Italian Inflammatory Bowel Diseases centres, starting from $1^{\text {st }}$ of May 2015 and until $31^{\text {st }}$ of December 2020.

\section{Clinical Assessment}

Eligible patients included men and women at least 18 years of age, an established diagnosis of UC according to standard endoscopic and histological criteria [1] active disease, defined as a Mayo score $\geq 3$ points despite concomitant treatment. Patients with less than 2-month follow-up were excluded.

A shared common database was used to collect demographic and clinical data. Data collected at baseline were gender, age at diagnosis, smoking status, disease extension (according to the Montreal classification [24]), disease severity (assessed using Mayo score and Mayo subscore for endoscopy [25]), disease duration, previous immunosuppressive and infliximab therapies, concomitant medications at baseline, C-reactive Protein (CRP) and fecal calprotectin (FC) levels. Patients were clinically assessed at entry and at month 2, 3, 6, 9, 12, and then every 12 months.

The study protocol conforms to the ethical guidelines of the 1975 Declaration of Helsinki as reflected in a priori approval by the institution's human research committee. The study was conducted according to the clinical practice guidelines. All patients gave written informed consent before they underwent endoscopy and s.c. GOL treatment.

\section{Study Treatment}

All patients were eligible for injection of s.c. GOL after exclusion of active hepatitis B virus infection, active cytomegalovirus infection, and tuberculosis.

Induction dose of GOL was $200 \mathrm{mg}$ at week $0,100 \mathrm{mg}$ at week 2 , and then 100 or $50 \mathrm{mg}$ at week 6 and then every four weeks according to the body weight.

The need for treatment discontinuation or dose escalation was left to the investigators' judgement, as well as concomitant medications including oral and topical aminosalicylates, steroids and immunosuppressants.

\section{Endoscopy}

Ileo-colonoscopy was retrospectively classified according to the Mayo subscore for endoscopy [25]. We enrolled only patients that had an endoscopic assessment before starting GOL therapy.

\section{Endpoints}

Primary endpoints were induction of remission, defined as Mayo score $\leq 2$, during the follow-up and maintenance of remission, defined as Mayo score $\leq 2$, during the follow-up.
Secondary endpoints were: 1) clinical response, defined as a reduction of at least 2 points in the Mayo score during follow-up (if blood in stool was present, it had to be reduced by at least one point; 2) mucosal healing $(\mathrm{MH})$, defined as Mayo subscore for endoscopy $\leq 1$, at any time during the follow-up; 3) steroid-free remission during the study; 4) need of colectomy; 5) adverse events (AEs) incidence during treatment. The AEs were subdivided as early (occurring during infusion), and late (occurring at least one week after the infusion) events and graded as mild (not requiring to stop treatment) and severe (requiring to stop treatment). Occurrence of opportunistic infections was also considered as an AE. It was defined as any infection caused by microorganisms that have limited pathogenic capacity under normal circumstances, but that have been able to cause disease because of the predisposing effect of the treatment [26];6) discontinuation of treatment, due to primary failure (defined as failure in reaching remission/ clinical response at any time of treatment), or secondary failure (defined as loss of remission/clinical response after reaching it under treatment), or due to side-effects; 7) escalation of treatment, due to primary loss of remission/clinical response; 8) Behaviour of CRP and FC during the follow-up.

\section{Statistics}

Data were analyzed using MedCalc ${ }^{\circledR}$ Release 14.8.1. The characteristics of the study group were analyzed as median with interquartile range (IQR) for continuous non-parametric variables and as a percentage for categorical variables. Factors associated with clinical remission were analyzed by means of Kaplan Meier method with hazard ratio (HR) and Logrank test. The Friedman test was used to investigate any change of C-reactive protein (CRP) and fecal calprotectin (FC) levels during follow-up. $\mathrm{P}$ values $<0.05$ were considered statistically significant.

\section{RESULTS}

One hundred and seventy-eight patients were enrolled and followed up for a median (IQR) time of 9 (3-18) months (mean time follow-up was 33.1 \pm 13 months). All patients were naïve to biologic therapy, and the clinical characteristics of the study group and the indication for s.c. GOL treatment are shown in Table I.

\section{Primary Endpoints}

Clinical remission was achieved in 57 (32.1\%) patients: these patients continued with GOL, but only 6 patients (3.4\%) were still under remission with GOL at the 42nd month of follow-up (Fig. 1). Clinical remission showed no correlation with gender, age, disease duration, baseline Mayo partial score, smoking, steroid therapy, immunomodulators treatment, Mayo subscore for endoscopy, FC and CRP levels at entry (Table II).

\section{Secondary Endpoints}

Six out 57 patients (10.8\%) needed dose escalation (from 50 to $100 \mathrm{mg}$ every 4 weeks) for loss of response, in order to maintain remission. Clinical response during the follow-up occurred in $64(36.4 \%)$ patients. 
Table I. Demographics, disease characteristics, and concomitant medications

\begin{tabular}{ll}
\hline Characteristics & \\
\hline Male gender, n (\%) & $92(51.7)$ \\
Median (IQR) age, years & $44.5(35-54)$ \\
Median (IQR) disease duration, years & $8(3-15)$ \\
Indication for therapy & \\
$\quad$ Steroid-dependency & $55(30.9)$ \\
$\quad$ Steroid-resistance & $111(62.4)$ \\
$\quad$ Switch for failure & $12(6.7)$ \\
Extent of disease & \\
$\quad$ Distal colitis & $15(8.4)$ \\
$\quad$ Left-sided colitis & $70(39.3)$ \\
$\quad$ Pancolitis & $93(52.2)$ \\
Median (IQR) Mayo score & $7(5-8)$ \\
Smoking, n (\%) & $16(9.0)$ \\
Patients receiving corticosteroids, n (\%) & $127(71.3)$ \\
Patients receiving immunomodulatory drugs, n (\%) & $34(19.1)$ \\
Median (IQR) Mayo subscore for endoscopy & $3(2-3)$ \\
Median (IQR) CRP (mg/L) & $8.0(5.0-21.0)$ \\
Median (IQR) calprotectin (mcg/g) & $374.5(200.0-1030.5)$ \\
\hline $\begin{array}{l}\text { Data are given as number (percentage) of patients } \\
\text { interquartile range). CRP: C-reactive protein. }\end{array}$ & \\
& \\
\hline &
\end{tabular}

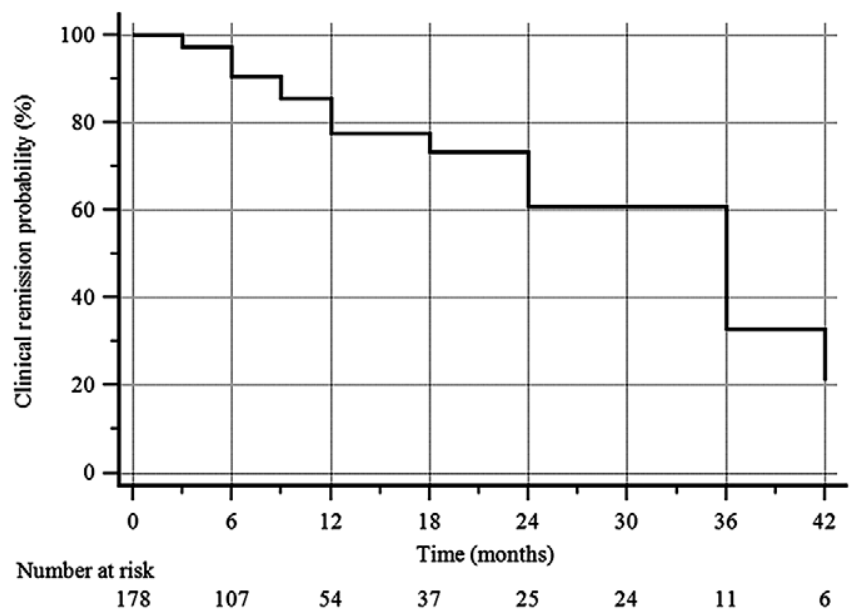

Fig. 1. Overall clinical remission maintenance during the follow-up Logrank test.

Primary failure occurred in $45(25.4 \%)$ patients, and secondary failure occurred in $58(32.6 \%)$ patients: 56 patients were treated with Vedolizumab, while 39 were treated with other anti-TNFa (25 with Infliximab and 14 with Adalimumab).

Colectomy was performed in $8(7.8 \%)$ patients, all of them having primary failure.

Steroid-free remission occurred in 23 (12.9\%) patients.

Ileo-colonoscopy was performed during the follow-up in 89 patients, and $\mathrm{MH}$ was achieved in 29 out of 89 (32.6\%) patients.

$\mathrm{CRP}$ and FC values during follow-up are reported in Fig. 2. A significant reduction of the level of the two parameters was present during follow-up with respect to baseline $(\mathrm{p}<0.0001)$.
Table II. Association of clinical characteristics with remission

\begin{tabular}{lcc}
\hline Variables & HR $(95 \%$ CI $)$ & $\mathrm{P}^{*}$ \\
\hline Sex, female & $1.109(0.658$ to 1.870$)$ & 0.659 \\
Age $>40$ years & $0.803(0.469$ to 1.375$)$ & 0.359 \\
Disease duration $>8$ years & $1.264(0.723$ to 2.210$)$ & 0.338 \\
Disease extension & Reference & - \\
$\quad$ Distal colitis & $0.608(0.238$ to 1.556$)$ & 0.779 \\
$\quad$ Left-sided colitis & $0.788(0.341$ to 1.821$)$ & 0.786 \\
$\quad$ Pancolitis & $0.903(0.533$ to 1.531$)$ & 0.305 \\
Mayo partial score $>7$ & $1.036(0.628$ to 1.708$)$ & 0.881 \\
No smoking & $1.391(0.819$ to 2.361$)$ & 0.146 \\
Patients not treated with steroids & $1.037(0.630$ to 1.712$)$ & 0.872 \\
Patients not treated with & & \\
immunomodulatory drugs & $0.940(0.501$ to 1.763$)$ & 0.834 \\
Mayo subscore for endoscopy $>3$ & $0.937(0.522$ to 1.681$)$ & 0.809 \\
CRP $>8$ (mg/L) & $1.166(0.651$ to 2.087$)$ & 0.562 \\
Fecal Calprotectin $>400$ ( $\mu$ gg $/ g)$ & & \\
\hline
\end{tabular}

HR: Hazard ratio; CRP: C-reactive protein. ${ }^{\star}$ Logrank test.

Adverse events occurred in 14 (7.9\%) patients (Table III), and caused withdrawal of the therapy in 8 cases.

\section{DISCUSSION}

We assessed the long-term performances of GOL in patients with UC in the real world. We also analyzed factors related to persistence. Our results showed that the rate of patients reaching remission was lower than $40 \%$. Moreover, the longterm persistence on GOL was very low, and just $3.4 \%$ of patients 

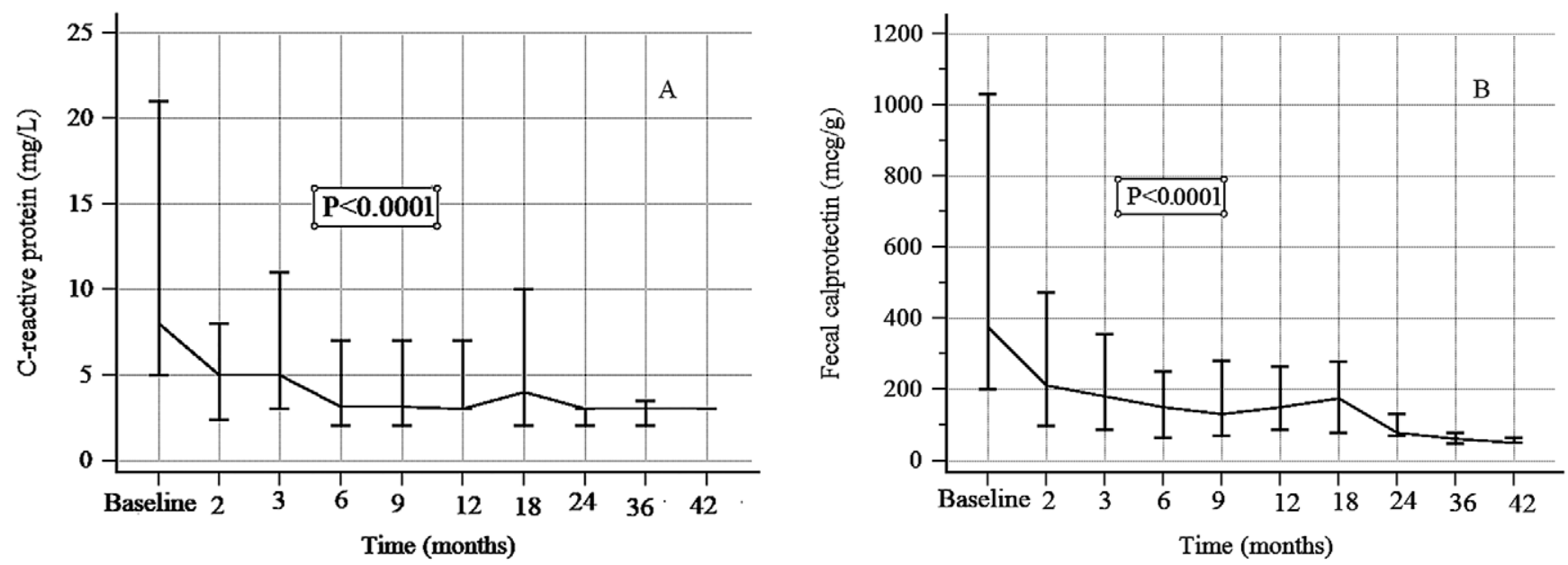

Fig. 2. C-reactive protein (A) and faecal calprotectin (B) values during follow-up. Data are expressed as median, interquartile range (error bars). Friedman test.

Table III. Adverse Events (AE)

\begin{tabular}{|c|c|}
\hline & $\begin{array}{l}\mathrm{N}(\%), \\
\text { (178 pts) }\end{array}$ \\
\hline Total Adverse Events (AE) & $14(7.9)$ \\
\hline Mild-Moderate AE & $6(3.3)$ \\
\hline Dermatitis in the site of injection & $3(1.7)$ \\
\hline Psoriasis & $1(0.6)$ \\
\hline Headache & $2(1.1)$ \\
\hline Severe AE & $8(4.5)$ \\
\hline Sistemic Allergy & $2(1.1)$ \\
\hline Severe Increased triglycerides & $1(0.6)$ \\
\hline Joint pain & $1(0.6)$ \\
\hline Pancreatitis & $1(0.6)$ \\
\hline Erythema nodosum & $1(0.6)$ \\
\hline Itching & $2(1.1)$ \\
\hline
\end{tabular}

were still on treatment at the $42^{\text {nd }}$ month of follow-up. These results were significantly different from those reported by the controlled studies. In the long-term extension of PURSUIT-M trial, $63 \%$ of patients remained on GOL treatment after 228 weeks of therapy [27]. But real-world studies showed different results. Looking at current real-life studies, focused on GOL persistence beyond 1-2 years, the remission maintenance occurred up to $27 \%$ at the 4 th year of treatment $[22,23]$.

Significantly, the unfavorable result of this study was obtained in patients naïve to biologic therapy, while in other studies the better performances were obtained in their own patients $[19,22,23]$. However, this advantage seems to drop at longer term follow-up [28]. Several factors were investigated in order to explain this result (disease extension, years of the disease, baseline disease severity), but we failed to find any significant link with this low remission rate. As expected, the final results were a high primary and secondary failure, leading to a large percentage of switch/swap to other biologics.

Why this unfavorable result occurs remains therefore unknown, however, some speculative hypotheses can be made. Firstly, real-life data found that anti-TNF antibodies work less in UC, both originator or biosimilars [21, 30, 31]; secondly several studies found GOL less effective than adalimumab and infliximab in UC patients [21, 29, 32]; thirdly, Jung et al. [33] found recently that, in UC, GOL initiators had a higher risk of non-persistence and switching than infliximab initiators, in particular when using corticosteroids. This last point could in part explain our results. Looking at the secondary points, we found that a very low percentage of patients reached a steroid-free remission, and similar results were obtained also by other Italian experiences $[18,19,21,23]$. This means that GOL is not really steroid sparing, increasing therefore, the risk of non-persistence and therefore switch/swap to other biologics. Finally, it is surprising to note that several Italian studies found that GOL failed to maintain the remission in a significant percentage of patients. For example, at week 52, Orlandini et al. [18] and Renna et al. [29] found a remission rate under GOL of 46-49\%, and Barberio et al. [21] found a remission rate of $38.7 \%$. Moreover, Pugliese et al. [23] found a remission rate of $22.5 \%$ at week 108 . The reaching and the maintenance of an adequate remission rate under GOL in the Italian UC population seems therefore difficult to reach, and more difficult to maintain. This could imply different genotypic characteristics of the UC Italian population, and further studies have to investigate this finding.

Looking at the other secondary points, we confirm the favorable safety profile of GOL. In fact, we recorded AEs in $7.9 \%$ of patients, lower than that reported by other long-term real-life studies [19-23]. However, it is hypothesized that the high rate of primary failure, with shorter follow-up, could influence these results. An interesting finding was that six patients needed dose escalation to maintain remission. Since the early dose intensification was found effective in nonresponders to induction treatment with GOL [34], the use of $100 \mathrm{mg}$ could be advise as standard treatment in all patients under maintenance with GOL because a high percentage of patients would benefit from both short-term efficacy and long-term persistence.

This study has strengths and limitations. The main strength lies in the large number of patients enrolled, fairly good length of follow-up, and the use of clinical scores for the evaluation of the disease. Regarding limitations, the first one is the retrospective design that does not permit to enroll patients having the same timing through the follow-up either for clinical or endoscopic follow-up. Furthermore, data on 
median follow-up was low due the high percentage of dropouts and not-responders. Although this could influence the long-term analysis, it further confirms that the long-term results with GOL are not satisfactory. Finally, drug serum levels and antidrug antibodies were not collected, because these analyses are not widely available in Italy, and their use in clinical practice is very limited. Meanwhile, despite all the aforementioned limitations, we believe that our data are sufficient to give adequate information about the long-term use of GOL in UC patients in Italy. In conclusion, this large real-life cohort study coming from Italian Inflammatory Bowel Diseases Centers has shown that GOL does not a have satisfactory rate of reaching and maintaining remission in UC patients. This result seems to be significantly different to that obtained in the rheumathoid arthtritis setting [35], once again advising caution in extrapolating the results from one disease to another, even if having a similar cytokine profile. Safety remains the best strength of this drug.

\section{Conflicts of interest: None to declare.}

Authors' affiliation: 1)Territorial Gastroenterology Service, ASL BAT, Andria; 2) Department of Medical and Surgical Sciences, Postgraduate School of Digestive Diseases, Catholic University, Rome; 3) Division of Gastroenterology, Brotzu Hospital, Cagliari; 4) Division of Gastroenterology, S. Camillo Hospital, Rome; 5) Division of Gastroenterology, Santa Caterina Novella Hospital, Galatina (LE); 6) Digestive Endoscopy Unit, San Matteo degli Infermi Hospital, Spoleto (PG); 7) Division of Gastroenterology, A.O. Ospedali Riuniti, Foggia; 8) Territorial Gastroenterology Service, PST Catanzaro Lido, Catanzaro; 9) Digestive Endoscopy Unit, AULSS7 Pedemontana, Santorso (VI); 10) Division of Digestive Endoscopy, S. Maria Goretti Hospital, Latina; 11) Department of Health Science, University of Catanzaro, Catanzaro; 12) Division of Gastroenterology, PTP Nuovo Regina Margherita, Roma; 13) Territorial Gastroenterology Service, ASL BA, Bari; 14) Ambulatory for IBD Treatment, Valle D'Itria Hospital, Martina Franca (TA); 15) Division of Gastroenterology, Ciaccio-Pugliese Hospital, Catanzaro; 16) Division of Gastroenterology, Belcolle Hospital, Viterbo; 17) Division of General Surgery, P. Colombo Hospital, ASL Roma 6, Velletri (Roma); 18) Gastroenterology Unit, Department of Biomedical and Clinical Sciences, L. Sacco University Hospital, Milan, Italy.

Authors' contribution: A.T. conceived and designed the study. A.T., G.Mocci., W.E., L.A., R.C., N.D.V., A.de M., R.F., A.F., G.F., T.L., R.L., F.L, A.P., G.P., S.R., R.S., L.S., C.Z., C.G., M.P., I.M.M.B, G.Maconi collected, analyzed and interpreted the data. A.T. G. Mocci, W.E., M.P., G.Maconi drafted the manuscript and revised it critically for important intellectual content. All the authors approved the final version of the paper.

\section{REFERENCES}

1. Dignass A, Eliakim R, Magro F, et al. Second European evidence-based consensus on the diagnosis and management of ulcerative colitis part 1: definitions and diagnosis. J Crohns Colitis 2012;6:965-990. doi:10.1016/j.crohns.2012.09.003
2. Dignass A, Lindsay JO, Sturm A, et al. Second European evidence-based consensus on the diagnosis and management of ulcerative colitis part 2: current management. J Crohns Colitis 2012;6:991-1030. doi:10.1016/j. crohns.2012.09.002

3. Lichtenstein GR, Abreu MT, Cohen R, Tremaine W; American Gastroenterological Association. American Gastroenterological Association Institute technical review on corticosteroids, immunomodulators, and infliximab in inflammatory bowel disease. Gastroenterology 2006;130:940-987. doi:10.1053/j.gastro.2006.01.048

4. Shealy DJ, Cai A, Staquet K, et al. Characterization of golimumab, a human monoclonal antibody specific for human tumor necrosis factor alpha. MAbs 2010;2:428-439. doi:10.4161/mabs.12304

5. Kay J, Matteson EL, Dasgupta B, et al. Golimumab in patients with active rheumatoid arthritis despite treatment with methotrexate:a randomized, double-blind, placebo-controlled, dose-ranging study. Arthritis Rheum 2008;58:964-975. doi:10.1002/art.23383

6. Keystone E, Genovese MC, Klareskog L, et al. Golimumab in patients with active rheumatoid arthritis despite methotrexate therapy: 52-week results of the GO-FORWARD study. Ann Rheum Dis 2010;69:11291135. doi:10.1136/ard.2009.116319

7. Emery P, Fleischmann RM, Moreland LW, et al. Golimumab, a human antitumor necrosis factor alpha monoclonal antibody, injected subcutaneously every four weeks in methotrexate-naive patients with active rheumatoid arthritis: twenty-four-week results of a phase III, multicenter, randomized, double-blind, placebo-controlled study of golimumab before methotrexate as first-line therapy for early-onset rheumatoid arthritis. Arthritis Rheum 2009;60:2272-2283. doi: 10.1002/art.24638

8. Smolen JS, Kay J, Doyle MK, et al. Golimumab in patients with active rheumatoid arthritis after treatment with tumour necrosis factor alpha inhibitors (GO-AFTER study): a multicentre, randomised, doubleblind, placebo-controlled, phase III trial. Lancet 2009;374:210-221. doi:10.1016/S0140-6736(09)60506-7

9. Braun J, Deodhar A, Inman RD, et al. Golimumab administered subcutaneously every 4 weeks in ankylosing spondylitis: 104-week results of the GO-RAISE study. Ann Rheum Dis 2012;71:661-667. doi:10.1136/ard.2011.154799

10. Kavanaugh A, van der Heijde D, McInnes IB, et al. Golimumab in psoriatic arthritis:one-year clinical efficacy, radiographic, and safety results from a phase III, randomized, placebo-controlled trial. Arthritis Rheum 2012;64:2504-2517. doi:10.1002/art.34436

11. Sandborn WJ, Feagan BG, Marano C, et al. Subcutaneous golimumab induces clinical response and remission in patients with moderateto-severe ulcerative colitis. Gastroenterology 2014;146:85-95. Doi:10.1053/j.gastro.2013.05.048

12. Sandborn WJ, Feagan BG, Marano C, et al; PURSUIT-Maintenance Study Group. Subcutaneous golimumab maintains clinical response in patients with moderate-to-severe ulcerative colitis. Gastroenterology 2014;146:96-109.e1. doi:10.1053/j.gastro.2013.06.010

13. Rutgeerts P, Feagan BG, Marano CW, et al. Randomised clinical trial:a placebo-controlled study of intravenous golimumab induction therapy for ulcerative colitis. Aliment Pharmacol Ther 2015;42:504-514. doi:10.1111/apt.13291

14. Bosca-Watts MM, Cortes X, Iborra M, et al. Short-term effectiveness of golimumab for ulcerative colitis:Observational multicenter study. World J Gastroenterol 2016;22:10432-10439. doi:10.3748/wjg.v22. i47.10432

15. Taxonera C, Rodríguez C, Bertoletti F, et al. Clinical Outcomes of Golimumab as First, Second or Third Anti-TNF Agent in 
Patients with Moderate-to-Severe Ulcerative Colitis. Inflamm Bowel Dis 2017;23:1394-1402. doi:10.1097/MIB.0000000000001144

16. Castro-Laria L, Argüelles-Arias F, García-Sánchez V, et al. Initial experience with golimumab in clinical practice for ulcerative colitis. Rev Esp Enferm Dig 2016;108:129-132. doi:10.17235/reed.2016.4068/2015

17. Tursi A, Allegretta L, Buccianti N, et al. Effectiveness and safety of golimumab in treating outpatient ulcerative colitis:a real-life prospective, multicentre, observational study in primary infammatory bowel diseases centers. J Gastrointestin Liver Dis 2017;26:239-244. doi:10.15403/jgld.2014.1121.263.trs

18. Orlandini B, Dragoni G, Variola A, et al. Clinical efficacy and safety of golimumab in biologically experienced and naive patients with active ulcerative colitis: A real-life experience from two Italian IBD centers. J Dig Dis 2018;19:468-474. doi:10.1111/1751-2980.12648

19. Bossa F, Biscaglia G, Valvano MR, et al. Real-Life Effectiveness and Safety of Golimumab and Its Predictors of Response in Patients with Ulcerative Colitis. Dig Dis Sci 2020;65:1767-1776. doi:10.1007/s10620019-05904-Z

20. Teich N, Grümmer H, Jörgensen E, et al. Golimumab in real-world practice in patients with ulcerative colitis:Twelve-month results. World J Gastroenterol 2020;26:2852-2863. doi:10.3748/wjg.v26.i21.2852

21. Barberio B, Zingone F, Frazzoni L, et al. Real-Life Comparison of Different Anti-TNF Biologic Therapies for Ulcerative Colitis Treatment: A Retrospective Cohort Study. Dig Dis 2021;39:16-24. doi:10.1159/000508865

22. Iborra M, García-Morales N, Rubio S, et al. Real-life experience with 4 years of golimumab persistence in ulcerative colitis patients. Sci Rep 2020;10:17774. doi:10.1038/s41598-020-73577-0

23. Pugliese D, Privitera G, Rogai F et al.; Italian Group for the Study of Inflammatory Bowel Disease (IG-IBD).Two-year effectiveness and safety of golimumab in ulcerative colitis: An IG-IBD study. United European Gastroenterol J 2021;9:102-109. doi:10.1177/2050640620974308

24. Satsangi J, Silverberg MS, Vermeire S, Colombel JF. The Montreal classification of inflammatory bowel disease: controversies, consensus, and implications. Gut 2006;55:749-753. doi:10.1136/gut.2005.082909

25. Schroeder KW, Tremaine WJ, Ilstrup DM. Coated oral 5-aminosalcylic acid therapy for mildly to moderately active ulcerative colitis. N Eng J Med 1987;317:1625-1629. doi:10.1056/NEJM198712243172603
26. Colombel JF, Sandborn WJ, Reinisch W, et al; SONIC Study Group. Infliximab, azathioprine, or combination therapy for Crohn's disease. N Engl J Med 2010;362:1383-1395. doi:10.1056/NEJMoa0904492

27. Reinisch W, Gibson PR, Sandborn WJ, et al. Long-term benefit of golimumab for patients with moderately to severely active ulcerative colitis: Results from the PURSUIT-maintenance extension. J Crohn Colitis 2018;12:1053-1066. doi:10.1093/ecco-jcc/jjy079

28. Castro-Laria L, Argüelles-Arias F, García-Sánchez V, et al. Initial experience with golimumab in clinical practice for ulcerative colitis. Rev Esp Enferm Dig 2016;108:129-132. doi:10.17235/reed.2016.4068/2015

29. Renna S, Mocciaro F, Ventimiglia M, et al. A real life comparison of the effectiveness of adalimumab and golimumab in moderate-to-severe ulcerative colitis, supported by propensity score analysis. Dig Liver Dis 2018;50:1292-1298. doi:10.1016/j.dld.2018.06.008

30. Tursi A, Mocci G, Lorenzetti R, et al. Long-term real-life efficacy and safety of infliximab and adalimumab in the treatment of inflammatory bowel diseases outpatients. Eur J Gastroenterol Hepatol 2021;33:670679. doi:10.1097/MEG.0000000000002087

31. Tursi A, Mocci G, Faggiani R, et al. Infliximab biosimilar CT-P13 is effective and safe in treating inflammatory bowel diseases:a real-life multicenter, observational study in Italian primary inflammatory bowel disease centers. Ann Gastroenterol 2019;32:392-399. doi:10.20524/aog.2019.0377

32. Renna S, Orlando E, Macaluso FS, et al. Letter: a prospective real life comparison of the efficacy of adalimumab vs. golimumab in moderate to severe ulcerative colitis. Aliment Pharmacol Ther 2016;44:310-311. doi:10.1111/apt.13692

33. Jung YS, Han M, Park S, Cheon JH. Biologic Use Patterns and Predictors for Non-persistence and Switching of Biologics in Patients with Inflammatory Bowel Disease: A Nationwide Population-Based Study. Dig Dis Sci 2020;65:1436-1444. doi:10.1007/s10620-019-05867-1

34. Philip G, Cornillie F, Adedokun JO, et al. Early dose optimization of golimumab in nonresponders to induction treatment for ulcerative colitis is effective and supported by pharmacokinetic data. J Crohn Colitis 2019;13:1257-1264. doi:10.1093/ecco-jcc/jjz052

35. Iannone F, Santo L, Anelli MG, et al. Golimumab in real-life settings: 2 Years drug survival and predictors of clinical outcomes in rheumatoid arthritis, spondyloarthritis, and psoriatic arthritis. Semin Arthritis Rheum 2017;47:108-114. doi:10.1016/j.semarthrit.2017.01.008 